\title{
Peiminine serves as an adriamycin chemosensitizer in gastric cancer by modulating the EGFR/FAK pathway
}

\author{
QIANQIAN TANG ${ }^{1,2}$, YUNFEI WANG $^{1}$, LANJING MA $^{3}$, MEILING DING $^{3}$, \\ TINGYU $\mathrm{LI}^{3}$, YONGZHAN NIE ${ }^{3}$ and ZHENGYI GU ${ }^{1}$

\begin{abstract}
${ }^{1}$ Key Laboratory of Xinjiang Uighur Medicine, Xinjiang Institute of Materia Medica, Urumqi 830004;
${ }^{3}$ State Key Laboratory of Cancer Biology and Xijing Hospital of Digestive Diseases, Xijing Hospital,

The Fourth Military Medical University, Xi'an, Shaanxi 710032, P.R. China
\end{abstract} \\ ${ }^{2}$ College of Pharmacy, Xinjiang Medical University, Urumqi, Xinjiang Uyghur Autonomous Region 830011;
}

Received June 12, 2017; Accepted December 1, 2017

DOI: $10.3892 / o r .2018 .6184$

\begin{abstract}
Gastric cancer (GC) is one of the most common malignancies of the digestive tract. Adriamycin (ADR) has been widely utilized in various chemotherapy regimens for treating GC, yet its long-term application may increase drug resistance resulting in treatment failure. Increasing evidence shows that bioactive natural products can be used as chemotherapeutic sensitizers that can significantly improve chemotherapy sensitivity. Peiminine (PMI) is a biologically active component extracted from Fritillaria walujewii Regel. Thus, in the present study, we aimed to investigate whether peiminine (PMI) alters the chemosensitivity of GC to adriamycin (ADR). GC cells were treated with ADR with or without PMI. MTT assay, flow cytometry and a nude mouse tumor xenograft model of SGC7901 cells were used to evaluate the chemosensitization activity of PMI combined with ADR. Western blotting was used to examine the expression of cyclin D1 and cleaved PARP. The RayBio ${ }^{\circledR}$ Human RTK phosphorylation antibody array kit was used to test the differential protein expression. Compared with the ADR group, PMI combined with ADR significantly suppressed cell proliferation and induced cell apoptosis in vitro. The growth curve and tumor weight of the tumor xenografts were significantly decreased in mice treated with the combination of PMI and ADR. However, the organs showed no obvious abnormality after treatment with PMI plus ADR. The expression of cyclin D1 was decreased and the level
\end{abstract}

Correspondence to: Professor Zhengyi Gu, Key Laboratory of Xinjiang Uighur Medicine, Xinjiang Institute of Materia Medica, 140 Xinhua South Road, Urumqi, Xinjiang Uyghur Autonomous Region 830004, P.R. China

E-mail: zhengyi087@126.com

Professor Yongzhan Nie, State Key Laboratory of Cancer Biology and Xijing Hospital of Digestive Diseases, Xijing Hospital, The Fourth Military Medical University, 127 West Changle Road, Xi'an, Shaanxi 710032, P.R. China

E-mail: yongznie@fmmu.edu.cn

Key words: peiminine, adriamycin, gastric cancer, chemosensitizer of cleaved PARP was increased after treatment with PMI and ADR. The expression of p-EGFR and p-FAK was downregulated in cells treated with PMI and ADR, and the validation of p-EGFR and p-FAK was in accordance with the result of the phosphorylation antibody array kit. PMI may serve as a new chemosensitizer by inhibiting the proliferation and inducing the apoptosis to enhance the chemotherapeutic drug sensitivity of ADR in GC.

\section{Introduction}

Gastric cancer (GC), one of the most common malignancies, is the third leading cause of cancer-related death worldwide $(1,2)$. To date, chemotherapeutic agents have been considered as a treatment option for various types of tumors. However, their clinical efficiency is hampered by cytotoxicity and chemotherapy resistance $(3,4)$.

Adriamycin (ADR), a cell cycle non-specific drug, shows a variety of therapeutic effects on tumors as it inhibits DNA and RNA synthesis. ADR and its derivatives have been widely utilized in various chemotherapy regimens for treating GC (5). Despite its obvious antitumor effects, it is reported to show marked toxicity. However, the toxicity is usually inconspicuous and before diagnosis of the adverse effects, the treatment may negatively affect various organs such as the brain, heart and kidneys (6). Moreover, its long-term application may increase drug resistence and result in treatment failure (7). Therefore, it is urgent to identify novel drugs with which to enhance the drug sensitivity of ADR and reduce its toxicity in clinical practice.

Increasing evidence shows that bioactive natural products can be used as chemotherapeutic sensitizers that can significantly improve chemotherapy sensitivity (8). In China, traditional Chinese medicine (TCM) has been used as a new origin for anticancer drugs for use as novel adjuvant chemotherapy treatments (NACTs) to improve the effectiveness of chemotherapy and to reduce side-effects and resistance of cancer chemotherapies (9). For example, gambogenic acid has been reported to increase the chemosensitivity of breast cancer cells to ADR via suppressing the PTEN/PI3K/AKT pathway (10). Meanwhile, Choi et al revealed that decursin found in Angelica gigas Nakai (AGN) could inhibit the proliferation of ADR-resistant ovarian 
cancer cells and induce apoptosis in the presence of ADR via blocking P-glycoprotein expression (11).

Peiminine (PMI) (Fig. 1A), is a biologically active component extracted from Fritillaria walujewii Regel of the Liliaceae family known as Xinjiang-Bei-Mu. Along with other alkaloids extracted from Fritillaria, PMI was reported to show biological effects as an antitussive and a relaxant of bronchial smooth muscle $(12,13)$. In addition, PMI was found to suppress colorectal cancer cell growth and cell proliferation by inducing autophagic cell death (14). In the present study, we focused on the sensitization effects of PMI on chemotherapy using ADR in the treatment of GC. Our data showed that PMI enhanced the chemotherapy sensitivity of GC to ADR, which suggested that the combination of PMI and ADR may be useful for treating human GC.

\section{Materials and methods}

Drugs. PMI (MW, $429.64 \mathrm{~g} / \mathrm{mol}$ ) with a purity of $>98 \%$ was obtained from the Xinjiang Institute of Materia Medica (Urumqi, China). It was solubilized in dimethyl sulfoxide (DMSO) before usage. ADR was purchased from Sigma-Aldrich (St. Louis, MO, USA).

Cell culture. Human GC cell lines SGC7901 (Academy of Military Medical Science, Beijing, China), SGC7901/ADR (human ADR-resistant cells) and SGC7901/VCR (human vincristine-resistant cells) (both from State Key Laboratory of Cancer Biology, Xi'an, China), and BGC823 (Academy of Military Medical Science) were maintained in our laboratory and cultured in RPMI-1640 medium (Gibco, Grand Island, NY, USA) supplemented with $10 \%$ fetal bovine serum (BI Biological Industries, Beit Haemek, Israel) and $1 \%$ penicillinstreptomycin sulphate at $37^{\circ} \mathrm{C}$ in a humidified air atmosphere containing $5 \% \mathrm{CO}_{2}$. To maintain the drug-resistance phenotype of the cell lines, the culture medium was supplemented with $0.5 \mu \mathrm{g} / \mathrm{ml}$ ADR for SGC7901/ADR cells and $1.0 \mu \mathrm{g} / \mathrm{ml}$ vincristine for SGC7901/VCR cells, respectively.

3-(4,5-Di-methyl-2-thiazolyl)-2,5-diphenyl-2H tetrazolium bromide (MTT) assay. MTT assay (Sigma-Aldrich) was performed to evaluate cell growth ability after PMI treatment using commercial kits according to the manufacturer's instructions, in order to select a non-toxic dose for the subsequent analysis. Briefly, GC cells (SGC7901, SGC7901/ADR, SGC7901/VCR and BGC823, 3×10 ${ }^{3}$ ) were diluted in $200 \mu \mathrm{l}$ of medium and plated in 96-well plates and incubated with PMI at different concentrations $(12.5,25,50,100,200$ and $400 \mu \mathrm{M})$. Cells were incubated for $72 \mathrm{~h}$, and then incubated for $4 \mathrm{~h}$ with $20 \mu \mathrm{l}(5.0 \mathrm{~g} / \mathrm{l}) \mathrm{MTT}$, followed by the addition of $150 \mu \mathrm{l}$ DMSO (both from Sigma-Aldrich) to each well to dissolve the crystals. The optical density (OD) values were read on a microplate reader (Bio-Rad 680; Bio-Rad, Hercules, CA, USA) at a wavelength of $490 \mathrm{~nm}$. Each experiment was performed in triplicate and repeated at least three times.

In vitro drug sensitivity assay. Drug sensitivity of GC cells to ADR combined with PMI was assessed by MTT assay as described above. Cells (SGC7901, SGC7901/ADR, SGC7901/VCR and BGC823) were treated with ADR in combination with PMI (non-toxic dosage) for $72 \mathrm{~h}$. Cells treated with ADR served as the control. The inhibition rates and the $\mathrm{IC}_{50}$ values were calculated. Each experiment was performed in triplicate and repeated three times.

In vitro apoptosis assay. Cell apoptosis in the four groups (e.g. DMSO, ADR, PMI and PMI+ ADR group) was detected using Dead Cell Apoptosis kit with Annexin V APC and SYTOX $^{\circledR}$ Green (Invitrogen-Molecular Probes, Eugene, OR, USA) for flow cytometry as previously described (15). All the tests were performed at least in triplicate.

In vivo drug sensitivity assay. Female BALB/c nude mice obtained from the Experimental Animal Center of the Fourth Military Medical University were used for the drug sensitivity assay. For the tumor challenge, SGC7901 cells $\left(2.0 \times 10^{6}\right)$ were subcutaneously injected into the left side of nude mice. Two weeks later, the animals were divided into the following groups: control $(n=5)$, received intraperitoneal (i.p.) injections of saline; ADR group $(n=5)$, receiving i.p. injections of ADR $(2.0 \mathrm{mg} / \mathrm{kg})$; PMI group $(\mathrm{n}=5)$, receiving i.p. injections of PMI $(2.5 \mathrm{mg} / \mathrm{kg})$; and ADR and PMI group, receiving a combination of ADR $(2.0 \mathrm{mg} / \mathrm{kg})$ and PMI $(2.5 \mathrm{mg} / \mathrm{kg})$. All groups were injected every two days during the treatment course. Tumor volume (V) was measured using a digital caliper every two days after chemotherapy according to the formula: $\mathrm{V}=\mathrm{LW}^{2} / 2$ (L, tumor length, W, tumor width).

Hematoxylin and eosin $(H \& E)$ staining and immunohistochemistry. After the sacrifice of the animals subjected to tumor challenge and treatment, the tumors were weighed, photographed and then fixed with $10 \%$ formaldehyde for $\mathrm{H} \& \mathrm{E}$ staining. Immunohistochemistry was performed for Ki67, as previously described (16). The organs were assessed using H\&E staining.

Human RTK phosphorylation antibody array. The RayBio ${ }^{\circledR}$ Human RTK phosphorylation antibody array kit (RayBiotech Inc., Norcross, GA, USA) was used for the Human RTK phosphorylation antibody array. Proteins were extracted from SGC7901 cells treated with ADR $(1.0 \mu \mathrm{M})$ and ADR $(1.0 \mu \mathrm{M})+\mathrm{PMI}(50.0 \mu \mathrm{M})$, respectively. Seventy-one proteins were tested according to the manufacturer's instructions. The images were scanned by ImageQuant LAS 4000 (GE Healthcare Corp., Piscataway, NJ, USA) with high resolution. The data were extracted and analyzed by the instrument analysis software.

Western blotting. SGC7901 cells treated with DMSO, ADR $(1.0 \mu \mathrm{M})$, PMI $(50.0 \mu \mathrm{M})$ and ADR $(1.0 \mu \mathrm{M})+\mathrm{PMI}$ $(50.0 \mu \mathrm{M})$, respectively, were homogenized in RIPA buffer (Beyotime, Jiangsu, China) containing protease inhibitors and phosphatase inhibitors (Roche, Basel, Switzerland). Total cell lysates were electrophoresed by sodium dodecyl sulfate-polyacrylamide gel electrophoresis (SDS-PAGE), and then transferred onto NC membranes (Sigma-Aldrich). The membranes were blocked in 5\% non-fat milk and incubated with primary antibody including rabbit anti-human cyclin D1 (\#2922), cl-PARP (\#5625), EGFR (\#4405), FAK (\#3285), p-FAK (Tyr397; \#3283), p-FAK (Tyr576; \#3281) (all from 

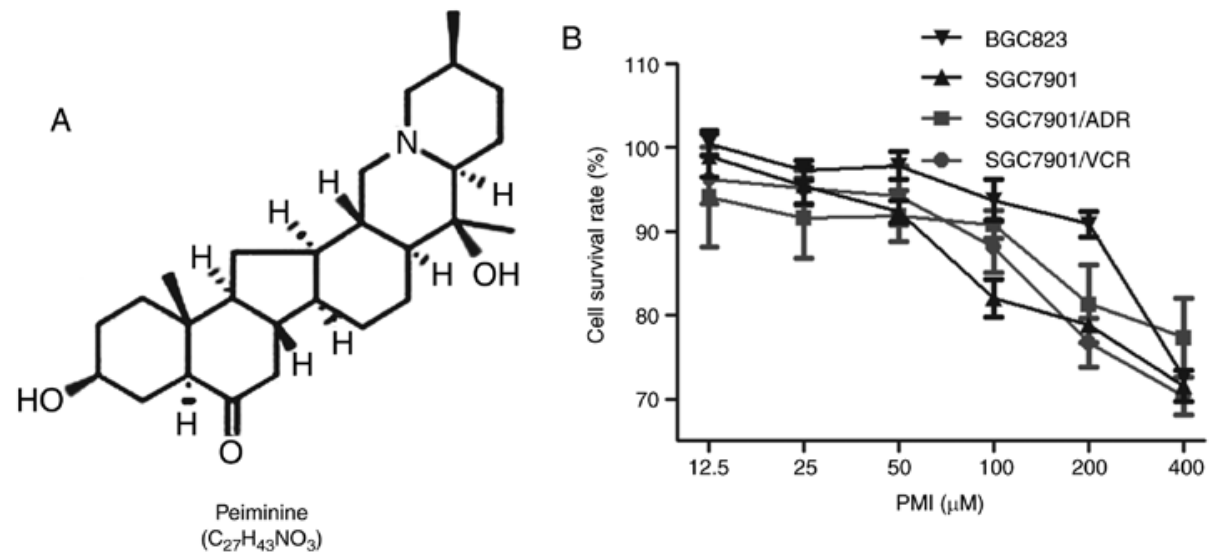

Figure 1. Cell viability of GC cells after PMI treatment was determined using an MTT assay. (A) Chemical structure of PMI. (B) Human GC cells were treated with various concentrations of PMI, and cell viability was evaluated using the MTT assay. A concentration of $50 \mu \mathrm{M}$ of PMI was used as the optimal concentration based on viability results for a non-toxic dose. GC, gastric cancer; PMI, peiminine.
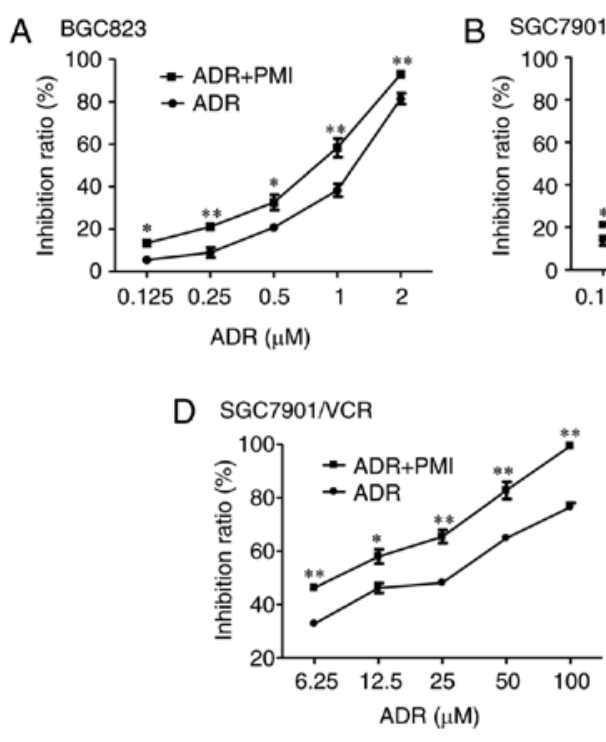
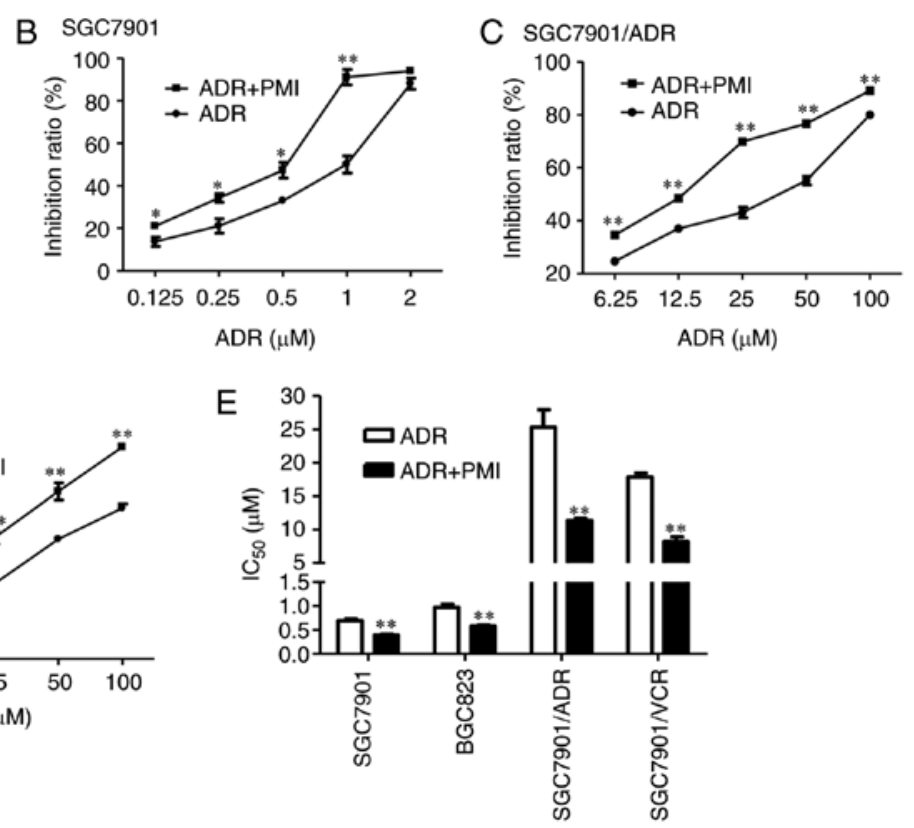

Figure 2. PMI enhances the activity of ADR in GC cells including (A) BGC823, (B) SGC7901, (C) SGC7901/ADR and (D) SGC7901/VCR cells. Cells treated with PMI $(50 \mu \mathrm{M})$ and various concentrations of ADR were assessed with an MTT assay. The cells treated with ADR alone were used as control ("P $<0.05$, $\left.{ }^{* *} \mathrm{P}<0.01\right)$. (E) The $\mathrm{IC}_{50}$ values of the four cell lines, which were treated with PMI and different concentrations of ADR. The cells treated with ADR alone were used as control ( $\left(\mathrm{P}<0.05,{ }^{* *} \mathrm{P}<0.01\right)$. ADR, adriamycin; GC, gastric cancer; PMI, peiminine.

Cell Signaling Technology, Inc, Beverly, MA, USA), p-EGFR (Tyr1068; \#ab32430; Abcam, Cambridge, MA, USA) (dilution ratio, 1:1,000; animal origins, rabbit anti-human), overnight at $4^{\circ} \mathrm{C}$, and then incubated with the peroxidase-conjugated goat anti-rabbit secondary antibody $(1: 1,000$; Abcam) for $1 \mathrm{~h}$ at room temperature. The same membrane was probed for $\beta$-actin for loading control. Blots were scanned by Molecular Imager ChemiDox XRS + Imaging System with Quantity one software (Bio-Rad).

Statistical analysis. All data are expressed as mean \pm standard error of mean. Two-tailed Student's t-test or an one-way ANOVA test was used to analyze the intergroup comparisons. Statistical tests were performed using SPSS 19.0 software (SPSS, Inc., Chicago, IL, USA). P $<0.05$ was considered to indicate a statistically significant result.

\section{Results}

Effects of PMI on the cell viability of the GC cell lines. To determine the cell cytotoxicity of PMI, we determined the cell viability using MTT assay, in which GC cell lines were treated with PMI at concentrations of 12.5, 25, 50, 100, 200 and $400 \mu \mathrm{M}$, respectively. Compared to the vehicle (DMSO), a high dose of PMI partly inhibited cell growth (Fig. 1B). A concentration of $50.0 \mu \mathrm{M}$ of PMI was used as the optimal concentration based on viability results for a non-toxic dose.

PMI enhances the chemotherapeutic drug sensitivity in GC cell lines. To investigate the chemotherapeutic drug sensitivity of PMI, we compared the efficiency of ADR combined with PMI and ADR alone in SGC7901, BGC823, SGC7901/ADR and SGC/7901/VCR cells, respectively. The combination of 
A

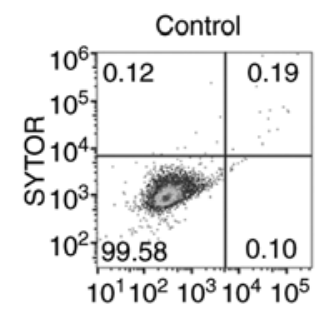

Annexin V

B

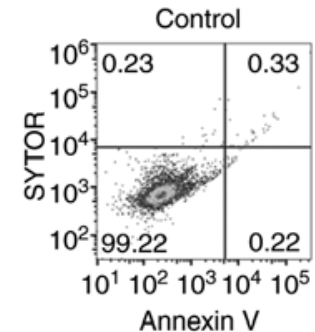

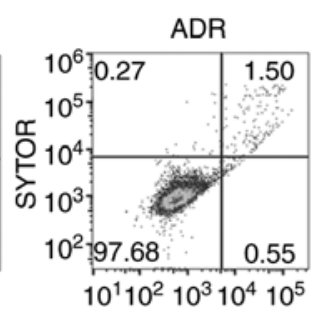

Annexin V

SGC7901

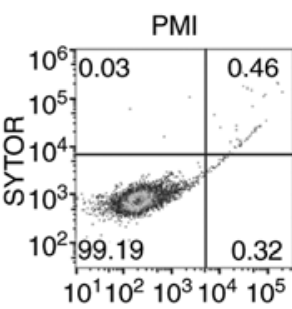

Annexin V

SGC7901/ADR

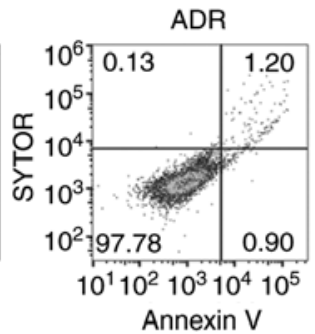

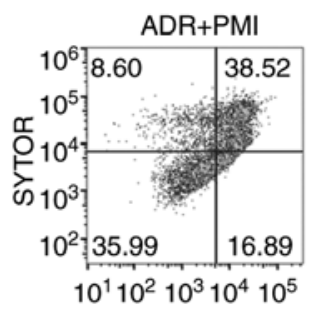

Annexin V

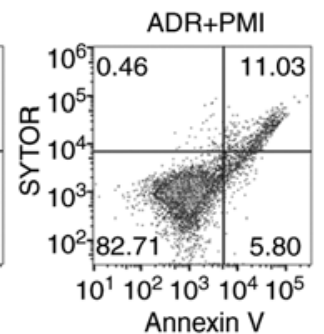

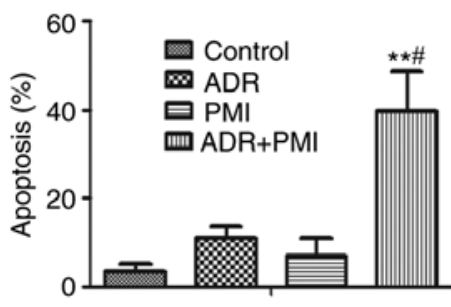

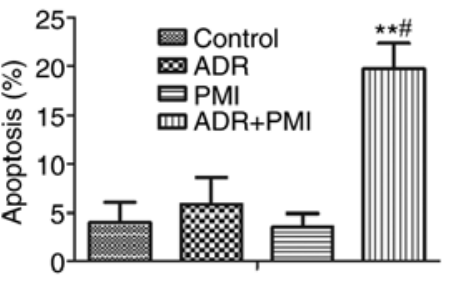

Figure 3. PMI combined with ADR induced the apoptosis in (A) SGC7901 and (B) SGC7901/ADR cells. The apoptotic rates of cells treated with DMSO (control), ADR (2.0 $\mu \mathrm{M}$ for SGC7901 cells; $60.0 \mu \mathrm{M}$ for SGC7901/ADR cells), PMI (50.0 $\mu \mathrm{M})$, and the combination of ADR and PMI were determined by flow cytometry; ${ }^{* *} \mathrm{P}<0.01$ compared with the control; ${ }^{*} \mathrm{P}<0.05$ compared with the ADR alone group. ADR, adriamycin; PMI, peiminine.

ADR and PMI conferred a significant toxic effect on these cells compared with those treated only using ADR (Fig. 2A-D), featured by significant inhibition rates $(\mathrm{P}<0.05)$ and a decrease in $\mathrm{IC}_{50}(\mathrm{P}<0.05$; Fig. 2E).

PMI combined with ADR induces the apoptosis of GC cells. Flow cytometry was carried out to analyze the apoptosis in SGC7901 and SGC7901/ADR cells after treatment with PMI, ADR or the combination of PMI and ADR. The results showed that the combination of PMI and ADR induced significant cell apoptosis compared with treatment with ADR alone $(\mathrm{P}<0.05$; Fig. 3). Thus, PMI enhanced the chemotherapy sensitivity of ADR via induction of apoptosis in the GC cells.

Chemosensitive effects of PMI in vivo. To investigate whether PMI enhances the ADR chemotherapeutic sensitivity of GC cells in vivo, we transplanted SGC7901 cells into nude mice. The tumor volume was significantly decreased in the PMI combined with ADR group compared with the control, PMI and ADR groups, respectively (Fig. 4A and B). This indicated that PMI enhanced the chemotherapeutic sensitivity of ADR. After sacrifice of the animals, the tumors were isolated, weighed and photographed (Fig. 4C). The combination of ADR and PMI induced significant inhibition activity of tumor volume compared with that of the control group and ADR group $(\mathrm{P}<0.01)$. Whereas, $\mathrm{PMI}$ induced no tumor inhibition activity compared to the control group (Fig. 4D).

Regarding the side-effects of the drug combination, no significant difference was noted in the body weight in the PMI group compared to the control group. However, a significant decrease was noted in the body weight in the ADR and ADR combined with PMI group compared to the control group (Fig. 4E). H\&E staining revealed that the combination treatment of ADR and PMI induced no pathological changes in the liver, kidney and heart compared to the control group (Fig. 4F). Immunohistochemistry showed that a high expression of Ki67 was exhibited in the control, while PMI combined with ADR decreased the expression of Ki67 in the tumor tissues (Fig. 4G). Taken together, we conclude that PMI enhanced ADR chemotherapy sensitivity in vivo.

PMI combined with ADR inhibits phosphorylation of receptor tyrosine kinases in GC cell lines. To further clarify how PMI acts as a regulatory factor in increasing the cell cytotoxicity of ADR, we examined the phosphorylation of receptor tyrosine kinases using a human RTK phosphorylation antibody array kit. SGC7901 cells were treated with ADR $(1.0 \mu \mathrm{M})$ or the combination of PMI $(50.0 \mu \mathrm{M})$ and ADR $(1.0 \mu \mathrm{M})$ for $24 \mathrm{~h}$. After normalization to the negative control (ADR), the phosphorylation of receptor tyrosine kinases was low in the drug combination group compared to the ADR group, including EGFR, FAK, Tyk2, ROS and LTK (Fig. 5A and B). Western blot assay showed that the expression levels of p-EGFR, EGFR, p-FAK and FAK were decreased in the drug combination group. However, the expression of cleaved PARP was upregulated and the expression of cyclin D1 was downregulated in SGC7901 cells treated with PMI combined with ADR (Fig. 5C and D).

\section{Discussion}

Reducing the side-effects of chemotherapy is a main strategy by which to improve the efficacy of chemotherapy. In China, traditional Chinese medicine (TCM) has been commonly used to improve cancer treatment efficiency in combination with chemotherapeutics serving as chemotherapeutic sensitizers. For example, increased attention has been paid to many TCM purification and monomers with low toxicity, high efficiency and safety in clinical practice.

Several TCM components have been used as chemotherapeutic sensitizers. For example, gambogenic acid was found to 
A

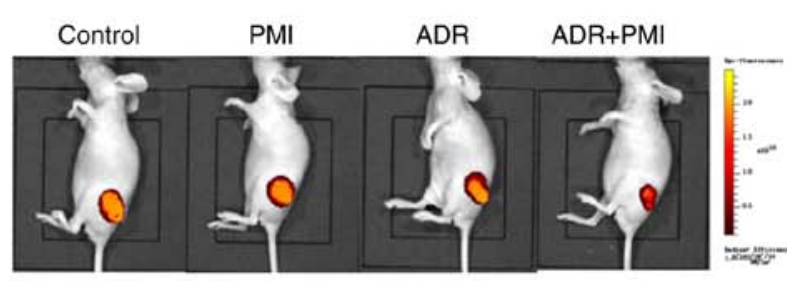

B

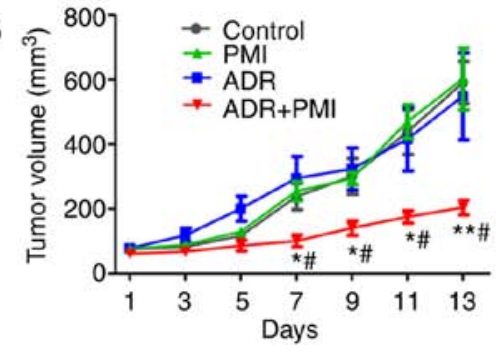

E

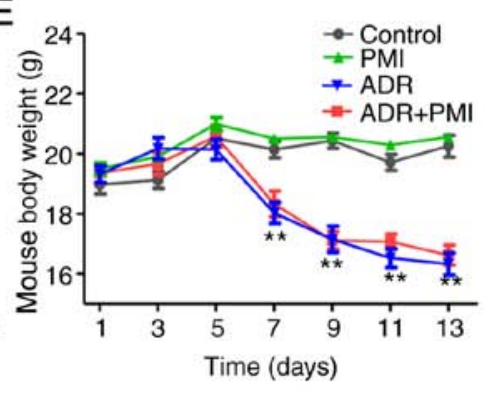

$\mathrm{F}$
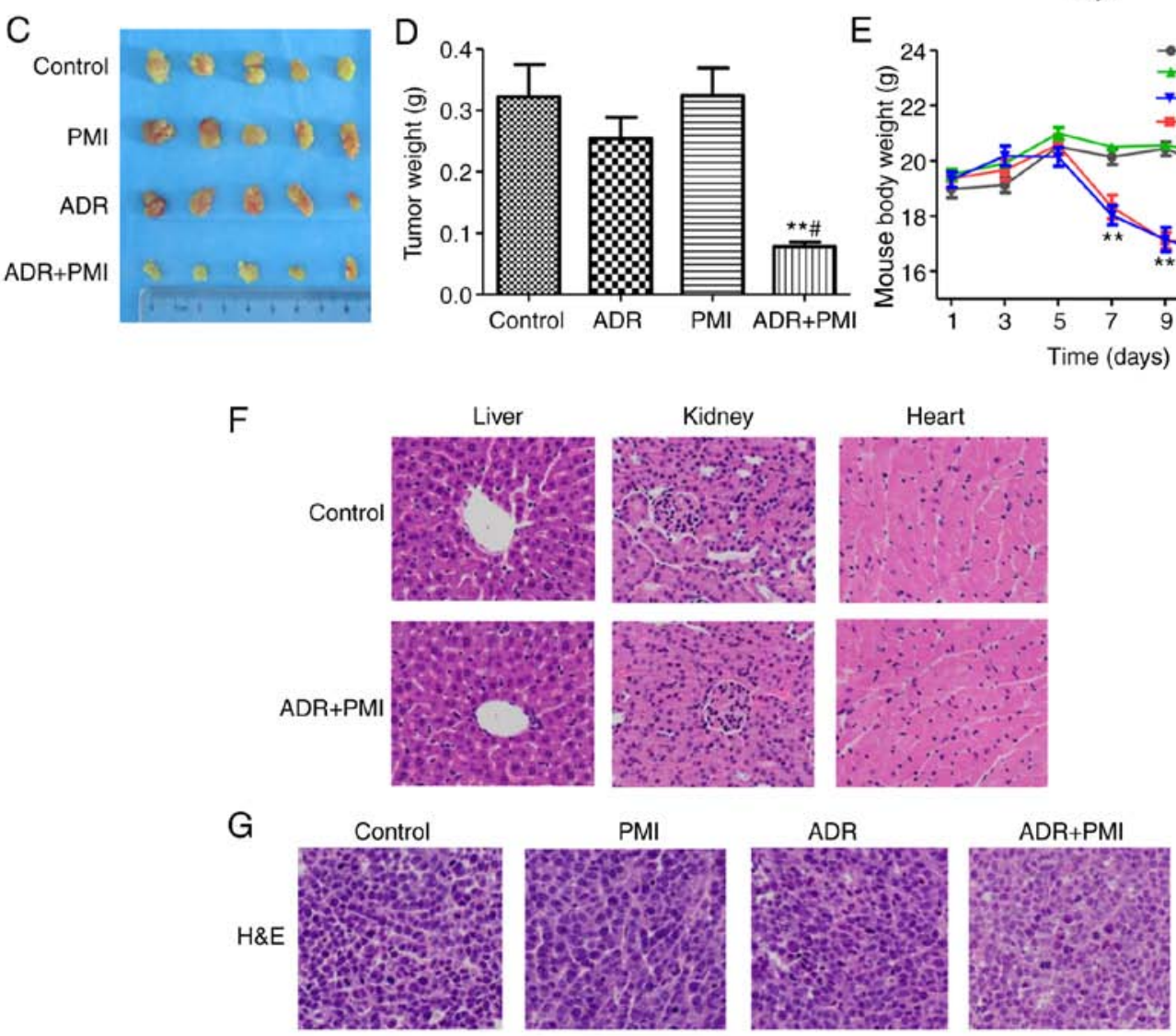

Ki67
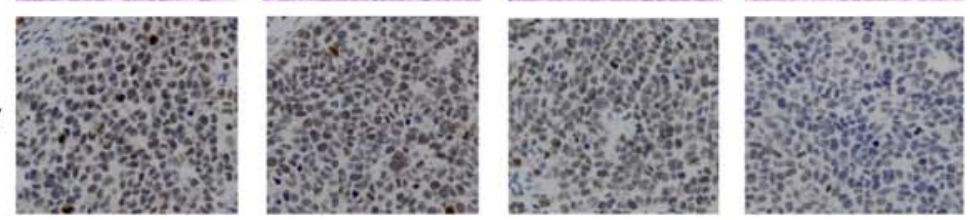

Figure 4. PMI combined with ADR suppresses tumor growth in vivo. (A) Images were captured using a living image system before sacrifice of the mice. (B) PMI combined with ADR contributed to the decrease in tumor growth. (C) Macroscopic appearance of the tumors in each group of mice at the end of the treatment period. (D) PMI combined with ADR induced a significant decrease in tumor weight. (E) The body weight of the PMI-treated mice did not differ from that of the saline controls, whereas ADR treatment reduced the mouse body weight. (F) H\&E staining indicated that no obvious organ injury occurred after PMI combined with ADR treatment. (G) The combination of PMI and ADR inhibited the expression of Ki67. Magnification, $\mathrm{x} 20$. ${ }^{*} \mathrm{P}<0.05,{ }^{* * *} \mathrm{P}<0.01$ compared with the control; " $\mathrm{P}<0.05$ compared with the ADR alone group. ADR, adriamycin; PMI, peiminine.

increase the chemosensitivity of breast cancer cells to adriamycin (ADR) by suppression of the PTEN/PI3K/AKT pathway leading to the apoptosis of MCF-7/ADR cells (10). Meanwhile, dioscin increased ADR chemosensitivity as it downregulated MDR1 expression by inhibiting the NF-kB signaling pathway in MCF-7/ADR cells (17). Quercetin was reported to enhance the sensitivity of breast cancer cells to doxirubicin by downregulating p-Akt expression arising from increased expression of PTEN (18). Cryptotanshinone enhanced the anticancer activity of doxirubicin in gastric cancer (GC) cells via STAT3 inactivation and suppression of STAT3-regulated antiapoptotic gene expression (19). Taken together, these agents serve as adriamycin sensitizers. The aim of the present study was to investigate whether PMI at non-toxic doses enhances the sensitivity of GC to ADR chemotherapy without additional toxicity. Our data demonstrated that the combination of PMI and ADR reduced cell viability as revealed by MTT assay. Compared with the PMI or ADR group, flow cytometry showed that the combination of PMI and ADR caused a marked induction in the apoptosis of GC cells. For the in vivo drug sensitivity experiment, mice received a dose of $2.5 \mathrm{mg} / \mathrm{kg}$ PMI which enhanced the chemotherapy sensitivity of ADR. Compared with the control group, the combination of ADR and PMI inhibited the tumor weight by $65.84 \%$, while ADR could only 

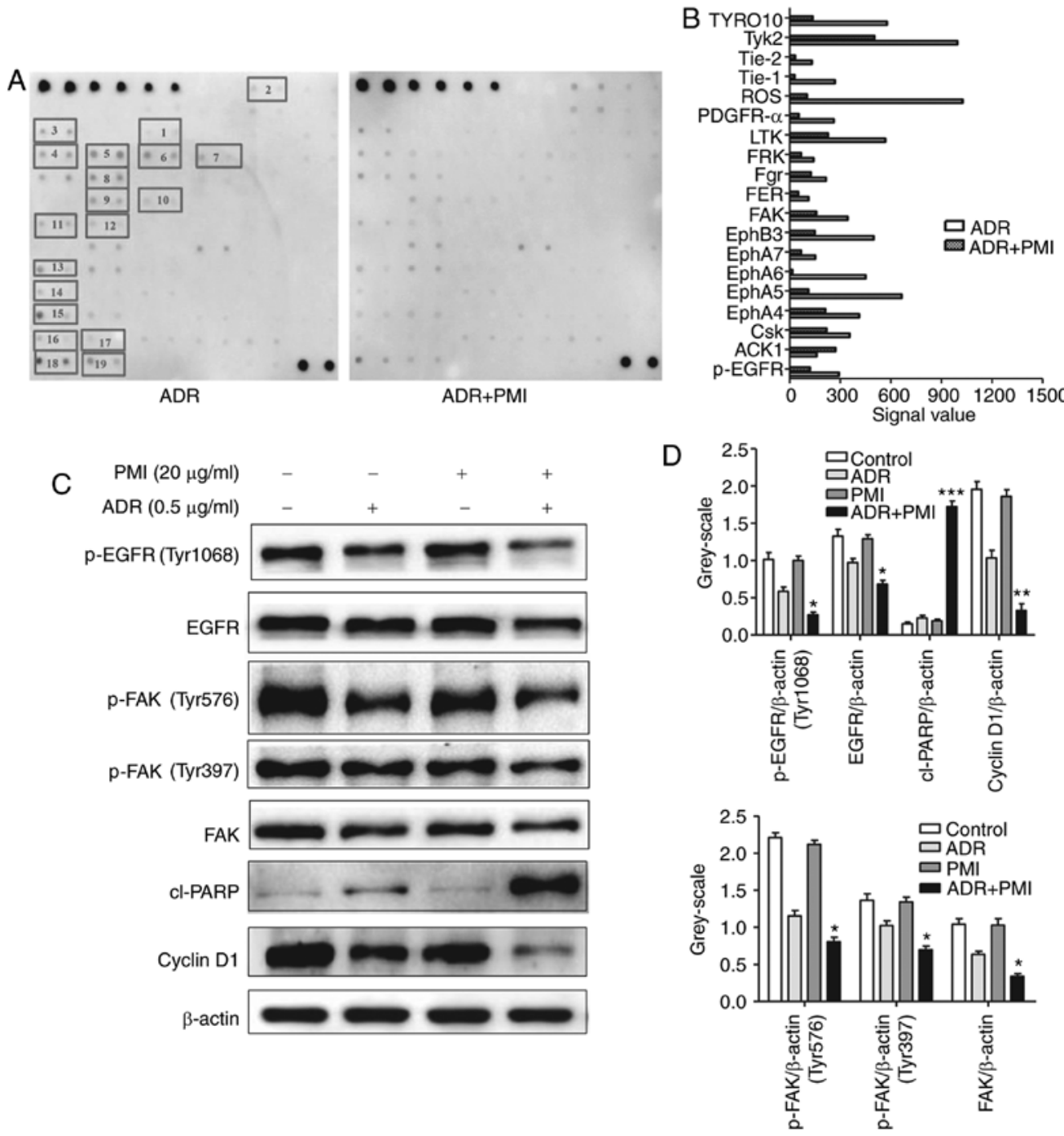

Figure 5. PMI combined with ADR suppresses the phosphorylation of receptor tyrosine kinases in GC cell lines. (A and B) SGC7901 cells were treated with ADR $(1.0 \mu \mathrm{M})$ or ADR $(1.0 \mu \mathrm{M})$ combined with PMI $(50.0 \mu \mathrm{M})$ and proteins were detected by the RayBio ${ }^{\circledast}$ Human RTK phosphorylation antibody array kit (chemiluminescent readout). 1, p-EGFR; 2, ACK1; 3, CsK; 4, EphA4; 5, EphA5; 6, EphA6; 7, EphA7; 8, EphB3; 9, FAK; 10, FER; 11, Fgr; 12, FRK; 13, LTK; 14, PDGFR- $;$ 15, ROS; 16, Tie-1; 17, Tie-2; 18, TyK2; 19, TYRO10. (C and D) Expression levels of p-EGFR, EGFR, p-FAK, FAK, cyclin D1 and cleaved (cl)-PARP were detected using western blotting. $\beta$-actin was used as a reference control. ADR, adriamycin; PMI, peiminine; ${ }^{2} \mathrm{P}<0.05,{ }^{* *} \mathrm{P}<0.01$ compared with the ADR alone group.

cause a decrease of $24.22 \%$. However, H\&E staining indicated no obvious abnormality after PMI plus ADR treatment, which demonstrated that PMI could be used as a chemotherapeutic sensitizer for the treatment of GC.

The epidermal growth factor receptor (EGFR) gene, a member of the EGFR family, encodes a $170 \mathrm{kDa}$ transmembrane tyrosine kinase receptor (20). EGFR was found to be an independent predictor of poor prognosis as it was overexpressed in GC patients (21-23). Berberine effectively enhanced the activity of EGFR inhibitors (erlotinib and cetuximab) in vitro and in vivo in GC (24). The combination medication of $\beta$-elemene and gefitinib not only inhibited the survival and proliferation of glioblastoma multiforme cells via inhibition of the EGFR signaling pathway but also induced more distinct apoptosis and autophagy in the glioblastoma multiforme cells when compared with the gefitinib monotherapy (25). EGFR has been reported to be implicated in tumor progression and is also a crucial transmembrane signal transduction pathway for many solid tumors $(26,27)$. To the best of our knowledge, EGFR is activated by ligand binding and succeeding receptor heterodimerization or homodimerization, which results in autophosphorylation of tyrosine residues and binding of adaptor molecules such as shc, gab-1 to the cytoplasmic domain. Src/FAK pathway is directly activated by phosphorylated receptors (28). Src/FAK and EGFR act synergistically through mutual phosphorylation and activation. The activation of EGFR enhanced Src expression contributed to tumor sensitivity of the Src inhibitor in lung cancer (29). In the present study, ADR combined with PMI downregulated the expression of p-EGFR and p-FAK, respectively.

FAK is widely known as a main mediator of migration, invasion, proliferation and oncogenic transformation (30). Recently, it has been reported to be involved in the pathogenesis of cancer. FAK is translocated to focal contact sites and autophosphorylated at its Tyr397 residue, which then leads to recruitment of downstream pathways by interacting with Src family kinases, PI3K, GRB7 and other signaling molecules (31). Increasing circumstantial evidence indicates that FAK overexpression contributes to the development of human maligancies, and has been acknowledged as an independent prognostic factor for 
ovarian (32), colon (33), human osteosarcoma (34) and GC (35). All these findings suggest that FAK plays an important role in cancer cell activity and disease progression. In the present study, PMI combined with ADR induced the downregulation of cyclin D1, p-EGFR and p-FAK. Additionally, the expression of cleaved-PARP was increased. These findings suggest the hypothesis that the sensitization effect of chemotherapy by PMI may involve the EGFR/FAK pathway.

In conclusion, the results demonstrated that PMI combined with ADR is an effective therapeutic strategy for the treatment of GC by inhibiting proliferation and inducing apoptosis. Further studies are required to understand the molecular mechanism of whether PMI contributes to the sensitization effect of chemotherapy by modulating the expression of EGFR or the cellular and subsequent inhibition of downstream FAK.

\section{Acknowledgements}

The present study was supported by the Key Laboratory Program of Xinjiang Autonomous Region (no. 2014KL006).

\section{References}

1. Van Cutsem E, Sagaert X, Topal B, Haustermans K and Prenen H: Gastric cancer. Lancet 388: 2654-2664, 2016.

2. Torre LA, Bray F, Siegel RL, Ferlay J, Lortet-Tieulent J and Jemal A: Global cancer statistics, 2012. CA Cancer J Clin 65: 87-108, 2015.

3. Cepeda V, Fuertes MA, Castilla J, Alonso C, Quevedo C and Pérez JM: Biochemical mechanisms of cisplatin cytotoxicity. Anticancer Agents Med Chem 7: 3-18, 2007.

4. Shi WJ and Gao JB: Molecular mechanisms of chemoresistance in gastric cancer. World J Gastrointest Oncol 8: 673-681, 2016.

5. Liu H, Li N, Yao L, Jiang L, Bao G, Li J, Ma Q and Liu Z: Prediction of doxorubicin sensitivity in gastric cancers based on a set of novel markers. Oncol Rep 20: 963-969, 2008.

6. Tacar O, Sriamornsak P and Dass CR: Doxorubicin: An update on anticancer molecular action, toxicity and novel drug delivery systems. J Pharm Pharmacol 65: 157-170, 2013.

7. Eker B, Meissner R, Bertsch A, Mehta K and Renaud P: Label-free recognition of drug resistance via impedimetric screening of breast cancer cells. PLoS One 8: e57423, 2013.

8. Vinod BS, Maliekal TT and Anto RJ: Phytochemicals as chemosensitizers: From molecular mechanism to clinical significance. Antioxid Redox Signal 18: 1307-1348, 2013.

9. Chen ZF and Liang H: Progresses in TCM metal-based antitumour agents. Anticancer Agents Med Chem 10: 412-423, 2010

10. He Y, Ding J, Lin Y, Li J, Shi Y, Wang J, Zhu Y, Wang K and $\mathrm{Hu} \mathrm{X}$ : Gambogenic acid alters chemosensitivity of breast cancer cells to Adriamycin. BMC Complement Altern Med 15: 181, 2015

11. Choi HS, Cho SG, Kim MK, Kim MS, Moon SH, Kim IH and Ko SG: Decursin in Angelica gigas Nakai (AGN) enhances doxorubicin chemosensitivity in NCI/ADR-RES ovarian cancer cells via inhibition of P-glycoprotein expression. Phytother Res 30: 2020-2026, 2016.

12. Wang D, Wang S, Chen X, Xu X, Zhu J, Nie L and Long X: Antitussive, expectorant and anti-inflammatory activities of four alkaloids isolated from Bulbus of Fritillaria wabuensis. J Ethnopharmacol 139: 189-193, 2012.

13. Wang DD, Feng Y, Li Z, Zhang L, Wang S, Zhang CY, Wang XX and Liu ZY: In vitro and in vivo antitumor activity of Bulbus Fritillariae Cirrhosae and preliminary investigation of its mechanism. Nutr Cancer 66: 441-452, 2014.

14. Lyu Q, Tou F, Su H, Wu X, Chen X and Zheng Z: The natural product peiminine represses colorectal carcinoma tumor growth by inducing autophagic cell death. Biochem Biophys Res Commun 462: 38-45, 2015.

15. Wang H, Cai S, Ernstberger A, Bailey BJ, Wang MZ, Cai W, Goebel WS, Czader MB, Crean C, Suvannasankha A, et al: Temozolomide-mediated DNA methylation in human myeloid precursor cells: Differential involvement of intrinsic and extrinsic apoptotic pathways. Clin Cancer Res 19: 2699-2709, 2013.
16. Zhu Y, Yu F, Jiao Y, Feng J, Tang W, Yao H, Gong C, Chen J, $\mathrm{Su}$ F, Zhang Y and Song E: Reduced miR-128 in breast tumorinitiating cells induces chemotherapeutic resistance via Bmi-1 and ABCC5. Clin Cancer Res 17: 7105-7115, 2011.

17. Wang C, Huo X, Wang L, Meng Q, Liu Z, Liu Q, Sun H, Sun P, Peng $J$ and Liu K: Dioscin strengthens the efficiency of adriamycin in MCF-7 and MCF-7/ADR cells through autophagy induction: More than just down-regulation of MDR1. Sci Rep 6: 28403, 2016.

18. Li SZ, Qiao SF,Zhang JH and Li K: Quercetin increase the chemosensitivity of breast cancer cells to doxorubicin via PTEN/Akt pathway. Anticancer Agents Med Chem 15: 1185-1189, 2015.

19. Wang J, Zhang G, Dai C, Gao X, Wu J, Shen L, Chen Z and Liu P: Cryptotanshinone potentiates the antitumor effects of doxorubicin on gastric cancer cells via inhibition of STAT3 activity. J Int Med Res 45: 220-230, 2017.

20. $\mathrm{Xu} Y \mathrm{YH}, \mathrm{Richert} \mathrm{N}$, Ito $\mathrm{S}$, Merlino GT and Pastan I: Characterization of epidermal growth factor receptor gene expression in malignant and normal human cell lines. Proc Natl Acad Sci USA 81: 7308-7312, 1984.

21. Ichikawa W, Kurahashi I, Sakuramoto S, Katai H, Sano T, Imamura $\mathrm{H}$ and Sasako M; ACTS-GC Group: Impact of expression of human epidermal growth factor receptors EGFR and ERBB2 on survival in stage II/III gastric cancer. Clin Cancer Res 18: 5992-6000, 2012.

22. Galizia G, Lieto E, Orditura M, Castellano P, Mura AL, Imperatore V, Pinto M, Zamboli A, De Vita F and Ferraraccio F: Epidermal growth factor receptor (EGFR) expression is associated with a worse prognosis in gastric cancer patients undergoing curative surgery. World J Surg 31: 1458-1468, 2007.

23. Lieto E, Ferraraccio F, Orditura M, Castellano P, Mura AL, Pinto M, Zamboli A, De Vita F and Galizia G: Expression of vascular endothelial growth factor (VEGF) and epidermal growth factor receptor (EGFR) is an independent prognostic indicator of worse outcome in gastric cancer patients. Ann Surg Oncol 15: 69-79, 2008.

24. Wang J, Yang S, Cai X, Dong J, Chen Z, Wang R, Zhang S, Cao H, Lu D, Jin T, et al: Berberine inhibits EGFR signaling and enhances the antitumor effects of EGFR inhibitors in gastric cancer. Oncotarget 7: 76076-76086, 2016.

25. Mu L, Wang T, Chen Y, Tang X, Yuan Y and Zhao Y: $\beta$-Elemene enhances the efficacy of gefitinib on glioblastoma multiforme cells through the inhibition of the EGFR signaling pathway. Int J Oncol 49: 1427-1436, 2016.

26. Proto $C$, Imbimbo $M$, Gallucci R, Brissa A, Signorelli D, Vitali M, Macerelli M, Corrao G, Ganzinelli M, Greco FG, et al: Epidermal growth factor receptor tyrosine kinase inhibitors for the treatment of central nervous system metastases from non-small cell lung cancer: The present and the future. Transl Lung Cancer Res 5: 563-578, 2016.

27. Brand TM, Iida M and Wheeler DL: Molecular mechanisms of resistance to the EGFR monoclonal antibody cetuximab. Cancer Biol Ther 11: 777-792, 2011.

28. Ayyappan S, Prabhakar D and Sharma N: Epidermal growth factor receptor (EGFR)-targeted therapies in esophagogastric cancer. Anticancer Res 33: 4139-4155, 2013.

29. Leung EL, Tam IY, Tin VP, Chua DT, Sihoe AD, Cheng LC, Ho JC, Chung LP and Wong MP: SRC promotes survival and invasion of lung cancers with epidermal growth factor receptor abnormalities and is a potential candidate for molecular-targeted therapy. Mol Cancer Res 7: 923-932, 2009.

30. Schwock J, Dhani N and Hedley DW: Targeting focal adhesion kinase signaling in tumor growth and metastasis. Expert Opin Ther Targets 14: 77-94, 2010.

31. Schlaepfer DD, Mitra SK and Ilic D: Control of motile and invasive cell phenotypes by focal adhesion kinase. Biochim Biophys Acta 1692: 77-102, 2004.

32. Hao Z, Qian J and Yang J: Shikonin induces apoptosis and inhibits migration of ovarian carcinoma cells by inhibiting the phosphorylation of Src and FAK. Oncol Lett 9: 629-633, 2015.

33. Heffler M, Golubovskaya VM, Dunn KM and Cance W: Focal adhesion kinase autophosphorylation inhibition decreases colon cancer cell growth and enhances the efficacy of chemotherapy. Cancer Biol Ther 14: 761-772, 2013.

34. Ren K, Lu X, Yao N, Chen Y, Yang A, Chen H, Zhang J, Wu S, Shi X, Wang C, et al: Focal adhesion kinase overexpression and its impact on human osteosarcoma. Oncotarget 6: 31085-31103, 2015.

35. Lai IR, Chu PY, Lin HS, Liou JY, Jan YJ, Lee JC and Shen TL: Phosphorylation of focal adhesion kinase at Tyr397 in gastric carcinomas and its clinical significance. Am J Pathol 177: 1629-1637, 2010. 